\title{
Structural insights into enzymatic mechanism of methylenetetrahydrofolate reductase (MTHFR) from Sphingobium sp. SYK-6
}

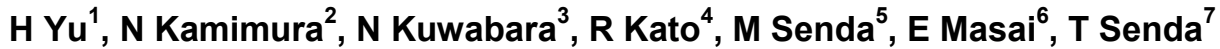 \\ 1 Structural Biology Research Center, Institute of Materials Structure Science, High Energy \\ Accelerator Research Organization (KEK), Tsukuba, ${ }^{2}$ Department of Bioengineering, Nagaoka \\ University of Technology, Nagaoka, ${ }^{3}$ SBRC, IMSS, KEK, ${ }^{4}$ Nagaoka University of Technology, ${ }^{5}$ High \\ Energy Accelerator Research Organization (KEK), Tsukuba, ${ }^{6}$ Department of Bioengineering, \\ Nagaoka University of Technology, ${ }^{7}$ Structural Bio Research Ctr Inst of Materials Structure, High \\ Energy Accelerator Research Org, Tsukuba \\ yuhong@post.kek.jp
}

Methylenetetrahydrofolate reductase (MTHFR) is a flavoprotein that involves nucleic acid and de novo methionine syntheses. MTHFR converts N5, N10-methylene-tetrahydrofolate (CH2-THF) to N5-methyl-tetrahydrofolate (CH3-THF) using NAD(P)H as an electron donor, and this enzyme also has the capability of catalyzing the reverse reaction using an artificial electron acceptor in vitro. Since most MTHFRs show a significantly high enzymatic activity for $\mathrm{NAD}(\mathrm{P}) \mathrm{H}$, it has been considered that MTHFR only converts $\mathrm{CH} 2-\mathrm{THF}$ to $\mathrm{CH} 3-\mathrm{THF}$ in cells. However, MTHFR from Sphingobium sp. SYK-6 (S6MTHFR), which utilizes lignin-derived aromatic compounds as a sole source of carbon energy, has been considered to catalyze the conversion from CH3-THF to CH2-THF in SYK-6. Although the cellular $\mathrm{NAD}(\mathrm{P}) \mathrm{H}$ concentration is much higher than CH3-THF, the opposite catalytic reaction by S6MTHFR suggests a different substrate specificity from those of typical MTHFRs. Since S6MTHFR shows similarity to other MTHFRs on the sequences, we speculated that structural changes of S6MTHFR in the process of evolution makes S6MTHFR prefer to react with CH3-THF rather than NAD(P)H. Therefore, we initiated to characterize this enzyme by using X-ray crystallography and biochemistry. In this study, we overexpressed S6MTHFR in Escherichia coli (E. coli) BL21, and purified by anion exchange and size exclusion chromatographies. Diffraction data for native SAD phasing were collected using X-ray wavelength of $2.7 \AA$ at BL1A of Photon Factory (KEK, Japan). To determine the structure, we limited the resolution to $2.60 \AA$ with 1.2 SigAno, 0.074 Rmerge, 51.1 multiplicity, and succeed in native SAD phasing by Crank2 in CCP4. After that, we determined the high-resolution apo and substrate $\mathrm{CH} 3$-THF complex structures using the molecular replacement method at $1.5 \AA$ and $1.8 \AA$ resolutions, respectively. The final Rwork/Rfree of the apo and substrate-complex structures were $0.17 / 0.20$ and $0.17 / 0.21$, respectively, by PHENIX.refine. By comparing to other MTHFR structures, S6MTHFR structures show significant differences. One prominent difference is that Cys219 interacts with the 2nd nitrogen atom of CH3-THF in the active site of S6MTHFR, however, such interaction has not been observed in E. coli MTHFR (EcMTHFR). Mutational analysis revealed that the Km value for CH3-THF of the C219A variant of S6MTHFR decreased 10 times compared to the wild type, suggesting Cys219 facilitates CH3THF binding. Furthermore, structure superposition of S6MTHFR with the NADH-EcMTHFR complex (PDB: 1zpt) suggested that Leu48 and Cys219 inhibit NADH binding to S6MTHFR. Indeed extremely low enzymatic activities of S6MTHFR were detected with NADH, and NADH hardly reduced FAD in S6MTHFR. Our structural and biochemical analysis revealed the molecular mechanism that S6MTHFR only catalyzes the conversion from CH3-THF to CH2-THF.

Acta Cryst. (2020). A76, a153 